\title{
VALIDATION OF TIMI AND GRACE SCORES IN ACUTE CORONARY SYNDROME (ACS) PATIENTS - A DEMOGRAPHIC STUDY IN A TERTIARY HOSPITAL OF NORTH KERALA
}

\author{
K. Kunhi Kannan1, Muhammed Mushthaque P2
}

${ }_{1}^{1}$ Associate Professor, Department of Medicine, Kannur Medical College, Anjarakandy, Kannur, Kerala. ${ }^{2}$ Associate Professor, Department of Medicine, Kannur Medical College, Anjarakandy, Kannur, Kerala.

\section{ABSTRACT}

\section{BACKGROUND}

Though the aetiology and pathophysiology of Acute Coronary Syndrome (ACS) is same whether the patients have ST segment elevation or not, but they form a heterogeneous group with variable short- and long-term prognoses. Early differentiation of these patients would help in better treatment and followup.

The aim of this study is to conduct a Demographic study of patients with ACS and validating them with both Thrombolysis in Myocardial Infarction (TIMI) and Global Registry of Acute Coronary Events (GRACE).

\section{MATERIALS AND METHODS}

76 patients with ACS admitted to the Department of Medicine through the casualty were included in the study. Demographic details were recorded. The final outcome of STEMI and UA/NSTEMI were compared.

\section{RESULTS}

Among 76 patients, 37 (46.68\%) with STEMI and 39 (51.31\%) with UA/NSTEMI were included. The comparable mean age among these two groups were $55 \pm 3.76$ and $57 \pm 4.12$ years respectively. Among the STEMI group, males were $78 \%$ and $64 \%$ in UA/NSTEMI group. Familial tendency was in $27.02 \%$ in STEMI compared to $23.07 \%$ in UA/NSTEMI. History of smoking was in $59 \%$ and $41 \%$ respectively. The UA/NSTEMI group had history of previous ischaemia in 73\%, unlike 27\% in STEMI group. Similarly hypertension was in $48.71 \%$ of UA/NSTEMI and in $37.83 \%$ of STEMI patients; Diabetes (29.72\% and 38.46\%). STEMI group patients had higher in-hospital MACE (35.13\% unlike UA/NSTEMI patients with 20.51\%). A higher 30-day all-cause mortality rate was $5.40 \%$ and $0 \%$ respectively and recurrent non-fatal MI of $2.70 \%$ and $0 \%$.

\section{CONCLUSION}

The age of occurrence of ACS is a little lower in these parts of India sharing common risk factors. Among ACS patients, STEMI group had bad in-hospital outcome and total 30-day outcome.

\section{KEYWORDS}

Acute Coronary Syndromes; Demographics; Risk Factor Scores; STEMI, TIMI; GRACE; Prognosis.

HOW TO CITE THIS ARTICLE: Kannan KK, Mushthaque MP. Validation of TIMI and GRACE scores in acute coronary syndrome (ACS) patients - a demographic study in a tertiary hospital of North Kerala. J. Evolution Med. Dent. Sci. 2017;6(8):615-619, DOI: 10.14260/Jemds/2017/132

\begin{abstract}
BACKGROUND
Even though the risk factors and pathophysiology are common for the patients of Acute Coronary Syndrome, either belonging to the STEMI and NSTEMI type, they form a heterogeneous group requiring early identification and treatment. The concept of risk assessment has become widespread in the last few years. ${ }^{1,2}$ Classifying the patient's risk factors in ACS helps one to identify high risk of death or recurrent ischaemic events, which might help us further to investigate and change the course of treatment. It also identifies low-risk patients, likely requiring early hospital discharge. It helps in better understanding of cost-benefit clinical resource utilisation like transfer to tertiary centres,
\end{abstract}

Financial or Other, Competing Interest: None.

Submission 06-01-2017, Peer Review 18-01-2017,

Acceptance 20-01-2017, Published 25-01-2017.

Corresponding Author:

Dr. K. Kunhi Kannan,

Associate Professor,

Department of Medicine,

Kannur Medical College,

Anjarakandy, Kannur, Kerala.

E-mail: ahmedmunirnet@gmail.com

DOI: $10.14260 /$ jemds $/ 2017 / 132$

(c) (i) $\ominus$ level of care, length of hospital stay and which pharmacological and interventional treatments should be used. ${ }^{2}$ The prognostic scores being used are derived from clinical trials such as TIMI (Thrombolysis in Myocardial Infarction). ${ }^{3,4}$ PURSUIT (Platelet glycoprotein IIb - IIIa in unstable angina, receptor suppression using Integrilin Therapy trial), ${ }^{5}$ and GUSTO (Global Utilisation of Streptokinase and t-PA for Occluded Coronary Arteries) ${ }^{6}$ scores. Some studies also use registries and cohort studies such as PREDICT (the Predicting Risk of Death in Cardiac Disease Tool), ${ }^{7}$ CCP (Cooperative Cardiovascular Project) 8 and GRACE (Global Registry of Acute Coronary Events) ${ }^{9}$ scores. STEMI and GRACE scores are calculated from initial clinical history and electrocardiographic and laboratory data collected on admission. These scores are very simple and practical at the bedside for risk assessments across a wide spectrum of patients with ACS.1,2 The classification helps the physicians' subjective risk selection of more aggressive therapies than would be chosen if patients were objectively classified by validated risk score models. Therefore, dedicated efforts to improve and implement risk classification may enhance the overall care process and resource utilisation. ${ }^{10}$ The present study aims at analysing the demographics of ACS patients admitted to Kannur Medical College, Anjarakandy, Kannur District, Kerala 
comparing ST Segment Elevation Myocardial Infarction (STEMI) with Unstable Angina/Non-ST Segment Elevation Myocardial Infarction (UA/NSTEMI) patients. To validate both TIMI and GRACE scores as tools to predict both inhospital Major Acute Cardiac Events (MACE) and 30-day allcause mortality.

\section{Inclusion Criteria}

Patients with history of coronary artery disease including Myocardial Infarction (MI) were included. Patients were assigned to types of STEMI and NSTEMI or unstable angina according to standard definitions were only included.

\section{Exclusion Criteria}

1. Patients with non-coronary causes of chest pain, i.e. trauma, gastritis or pericarditis were excluded.

2. Patients with acute dyspnoea from non-cardiac causes.

3. Patients with significant co-morbidity like advanced liver diseases, cerebrovascular diseases, malignancy and chronic lung diseases were excluded.

Total history taking was done and demographic details recorded by using a standard proforma. The management of ACS was according to the European guidelines. ${ }^{11}$ STEMI patients were re-perfused using streptokinase. Coronary angioplasty was done where the patient could afford. A standard 12-lead Electrocardiogram (ECG) record was recorded at a paper speed of $25 \mathrm{~mm} / \mathrm{s}$ and amplification of 10 $\mathrm{mm} / \mathrm{mV}$. Laboratory evaluation at the baseline included random blood sugar, serum lipid profile, serum urea and creatinine, creatine kinase, troponin and haemoglobin level on admission. The risk score assessment was done by using GRACE score according to risk model: age, history of congestive heart failure, history of MI, heart rate, systolic blood pressure on presentation, ST-segment deviation, initial serum creatinine and cardiac enzymes raised above the upper limit of normal for that laboratory. To record Major Acute Cardiac Events (MACE), the patients were reviewed daily until discharge and only the most serious events like death $>$ asystole $>$ cardiogenic shock $>$ pulmonary oedema $>$ serious arrhythmia $>$ recurrent non-fatal MI $>$ recurrent or refractory angina. MACE is reported according to the standard definitions.2,12 Statistical analysis of risk scores were calculated according to TIMI and GRACE risk score models. ${ }^{3,4,9}$ Data were analysed using Statistical Package for Social Sciences (SPSS) version 16.0.

\section{RESULTS}

The total number of patients included in the study was 76 . The patients were divided into two groups: 37 (46.68\%) with STEMI and 39 (51.31\%) with UA/NSTEMI were included. The age groups of both groups were similar in STEMI $55 \pm 3.76$ and $57 \pm 4.12$ in UA/NSTEMI. The highest percentage of patients was in the age group less than 65 years being $>70 \%$ for both groups and the lowest percentage of patients was in the age group 74 years being $10.81 \%$ for the STEMI group and $7.69 \%$ for the UA/NSTEMI group. The STEMI group had a significantly higher rate of male gender $(78.37 \%$ versus $64.10 \%$ of UA/NSTEMI), smoking (54.05\% versus $61.53 \%)$, diabetes $(29.72 \%$ versus $38.46 \%)$, dyslipidaemia $(39 \%$ versus $29 \%$ ) and family history of CAD (16\% versus $7 \%)$. The number of UA patients was $9(24.32 \%)$ and the number of
NSTEMI patients was 12 (30.76\%). Interestingly, the percentage of patients who had a previous history of ischaemia in the UA/NSTEMI group was $64.09 \%$ unlike $32.43 \%$ in STEMI group. The UA/NSTEMI group had a higher rate of hypertension ( $48.71 \%$ versus $37.83 \%$ ), diabetes (38.46\% versus $29.72 \%$ ) and previous coronary angiography (17.94\% versus $13.51 \%$ ). Patients with UA/NSTEMI had a longer duration since the first angina pain until presentation $(96 \pm 152 \mathrm{~h})$ compared with STEMI patients $(20 \pm 32 \mathrm{~h}$; p < 0.001). On the other hand, STEMI patients had more warning rest angina pain in the preceding $24 \mathrm{~h}(5 \pm 2)$ compared with UA/NSTEMI patients $(3 \pm 2 ; \mathrm{p}<0.001)$, (Table 1$)$.

\begin{tabular}{|c|c|c|c|}
\hline & $\begin{array}{c}\text { STEMI } \\
(\mathrm{NO}=37)\end{array}$ & $\begin{array}{c}\text { UA/NSTEMI } \\
(\mathrm{NO}=39)\end{array}$ & $\begin{array}{c}\text { p- } \\
\text { Value }\end{array}$ \\
\hline Male Gender & $29(78.37 \%)$ & $25(64.10 \%)$ & 0.02 \\
\hline \multicolumn{4}{|c|}{ Age in Years } \\
\hline$<65$ & $26(70.27 \%)$ & $28(71.79 \%)$ & \multirow{3}{*}{ NS } \\
\hline$\geqslant 65-<74$ & 7 (18.91\%) & $9(23.07 \%)$ & \\
\hline$\geqslant 74$ & $4(10.81 \%)$ & $3(7.69 \%)$ & \\
\hline Smoking & $20(54.05 \%)$ & $24(61.53 \%)$ & $<0.001$ \\
\hline Hypertension & $14(37.83 \%)$ & $19(48.71 \%)$ & $<0.001$ \\
\hline Diabetes mellitus & $11(29.72 \%)$ & $14(38.46 \%)$ & 0.006 \\
\hline Dyslipidaemia & $9(24.32 \%)$ & $12(30.76 \%)$ & 0.005 \\
\hline $\begin{array}{l}\text { Family history of } \\
\text { CAD }\end{array}$ & $10(27.02 \%)$ & $39(23.07 \%)$ & $<0.001$ \\
\hline Previous CA & $5(13.51 \%)$ & $7(17.94 \%)$ & $<0.001$ \\
\hline $\begin{array}{l}\text { First Angina until } \\
\text { presentation }\end{array}$ & $(96 \pm 152 h)$ & $(20 \pm 32 \mathrm{~h})$ & $<0.001$ \\
\hline \multicolumn{4}{|c|}{ History of Ischaemia } \\
\hline NO & $25(67.56 \%)$ & $13(35.33 \%)$ & \multirow{3}{*}{$<0.001$} \\
\hline STEMI & $8(21.62 \%)$ & $7(17.94 \%)$ & \\
\hline UA/NSTEMI & $4(10.81 \%)$ & $18(46.15 \%)$ & \\
\hline $\begin{array}{c}\text { Number of rest } \\
\text { angina in last } 24 \mathrm{~h}\end{array}$ & $5 \pm 2$ & $3 \pm 2$ & $<0.001$ \\
\hline \multicolumn{4}{|c|}{ Heart Rate (bpm) } \\
\hline$<60$ & $3(8.10 \%)$ & $5(12.82 \%)$ & \multirow{3}{*}{ NS } \\
\hline $60-100$ & $28(75.67 \%)$ & $31(79.48 \%)$ & \\
\hline$>100$ & $6(16.21 \%)$ & $3(7.69 \%)$ & \\
\hline \multicolumn{4}{|c|}{ Systolic Blood Pressure (mmHg) } \\
\hline$<120$ & $11(29.72 \%)$ & $8(20.51 \%)$ & \multirow{3}{*}{ NS } \\
\hline $120-139$ & $12(32.43 \%)$ & $14(35.89 \%)$ & \\
\hline$\geqslant 140$ & $14(37.83 \%)$ & $15(38.46 \%)$ & \\
\hline $\begin{array}{c}\text { Serum creatinine > } \\
120 \mathrm{mmol} / \mathrm{L}\end{array}$ & $8(21.62 \%)$ & $10(25.64 \%)$ & NS \\
\hline $\begin{array}{l}\text { Cardiogenic shock } \\
\text { on admission }\end{array}$ & $6(16.21 \%)$ & $3(7.69 \%)$ & $<0.001$ \\
\hline $\begin{array}{l}\text { ST-segment } \\
\text { deviation on } \\
\text { admission }\end{array}$ & $37(100 \%)$ & $25(64.10 \%)$ & $<0.001$ \\
\hline \multicolumn{4}{|c|}{$\begin{array}{c}\text { Table 1. Showing Differences between STEMI and } \\
\text { UA/NSTEMI Groups regarding Socio-Demographic } \\
\text { Variables and History of Ischaemia }\end{array}$} \\
\hline
\end{tabular}

(CA, Coronary Angiography; CABG, Coronary Artery Bypass Grafting; CAD, Coronary Artery Disease; STEMI, STElevation Myocardial Infarction; PAD, Peripheral Arterial Disease; UA/NSTEMI, Unstable Angina/Non-ST elevation Myocardial Infarction.) 
Regarding heart rate, both groups had the highest percentage of patients in the heart rate group $60-100 \mathrm{bpm}$ (75.67\% and $79.48 \%$, respectively). The major portion of the two groups had a systolic blood pressure of $140 \mathrm{mmHg}$, which was $37.83 \%$ in the STEMI group and $38.46 \%$ in the UA/NSTEMI group. The percentage of patients with elevated creatinine was almost equal in both groups being $25.64 \%$ in patients with UA/NSTEMI versus $21.62 \%$ in STEMI patients. Cardiogenic shock upon admission was significantly higher with STEMI (6 patients, 16.21\%) compared with UA/NSTEMI (three patients, $7.69 \%$ ). ST segment deviation was in $100 \%$ of STEMI and $64.10 \%$ of the UA/NSTEMI patients (Table 1 ).

There were $29.72 \%$ of patients (11) in the STEMI group, who had diabetes mellitus or hypertension and/or angina. On the other hand, a small percentage of patients had a systolic blood pressure $<100 \mathrm{mmHg}(13.51 \%)$ and heart rate $>100$ bpm (32.51\%). Patients who suffered from anterior STEMI or left bundle branch block represented the minor component of the STEMI group $(24.32 \%)$. In $75.67 \%$ of the patients, there was time for starting treatment more than 4 hours (Table 2).

Distribution of the clinical factors used in TIMI score calculation.

\begin{tabular}{|c|c|}
\hline TIMI for STEMI (No = 37) & No (\%) \\
\hline \multicolumn{2}{|l|}{ Age in years } \\
\hline$<65$ & $26(70.27 \%)$ \\
\hline$\geqslant 65-<74$ & $5(13.51 \%)$ \\
\hline$\geqslant 74$ & $6(7 \%)$ \\
\hline Diabetes, hypertension or angina & $11(29.72 \%)$ \\
\hline Systolic blood pressure $(<100 \mathrm{mmHg})$ & $5(13.51 \%)$ \\
\hline Heart rate (> $100 \mathrm{~b} / \mathrm{min})$ & $13(32.51 \%)$ \\
\hline Anterior ST elevation or LBBB & $9(24.32 \%)$ \\
\hline Angina start Time to treatment $(>4 \mathrm{~h})$ & $28(75.67 \%)$ \\
\hline TIMI for UA/NSTEMI $(\mathrm{NO}=39)$ & \\
\hline \multicolumn{2}{|l|}{ Diagnosis (UA or NSTEMI) } \\
\hline UA & $29(74.35 \%)$ \\
\hline NSTEMI & $11(28.20 \%)$ \\
\hline Age $(\geqslant 65$ years $)$ & $15(33.33 \%)$ \\
\hline$>3$ Risk factors for CAD & $33(84.61 \%)$ \\
\hline Aspirin use in the past 7 days & $30(76.92 \%)$ \\
\hline$>1$ episode rest Angina in $<24 \mathrm{~h}$ & $29(71.79 \%)$ \\
\hline ST-segment deviation & $32(82.05 \%)$ \\
\hline \multicolumn{2}{|c|}{$\begin{array}{c}\text { Table 2. Showing the Distribution of TIMI Score Factors } \\
\text { among Patients with STEMI and UA/STEMI }\end{array}$} \\
\hline
\end{tabular}

(CAD, Coronary Artery Disease; LBBB, Left Bundle Branch Block; NSTEMI, Non ST-Elevation Myocardial Infarction; STEMI, ST-Elevation Myocardial Infarction; TIMI, Thrombolysis In Myocardial Infarction; UA, Unstable Angina.) In the patients with UA/NSTEMI, approximately $74.35 \%$ were diagnosed with unstable angina, $33.33 \%$ with an age 65 years, $82.05 \%$ with an ST segment changes more than 3 risk factors in $84.61 \%$. Most patients $(71.79 \%)$ were found to have had severe anginal episodes. Aspirin was used in the last seven days by $70.3 \%$ of the patients and $80.6 \%$ were found to have $>3$ risk factors of coronary artery disease (Table 2).

In-hospital MACE was significantly higher among the STEMI patients $(51.35 \%)$ compared with the UA/NSTEMI (20.51\%). There was a significantly higher mortality, asystole, cardiogenic shock, serious arrhythmia, recurrent non-fatal MI with STEMI.

\begin{tabular}{|c|c|c|c|}
\hline & $\begin{array}{c}\text { STEMI } \\
(\mathbf{n = 3 7 )}\end{array}$ & $\begin{array}{c}\text { UA/NSTEMI } \\
(\mathbf{n = 3 9 )}\end{array}$ & P value \\
\hline MACE & $\mathbf{1 9}(\mathbf{5 1 . 3 5} \%)$ & $\mathbf{8}(\mathbf{2 0 . 5 1 \% )}$ & $\mathbf{0 . 0 0 1}$ \\
\hline In-hospital death & $2(5.40 \%)$ & 0 & $<0.001$ \\
\hline Asystole & $4(10.81 \%)$ & $1(2.56 \%)$ & 0.013 \\
\hline Cardiogenic shock & $6(16.21 \%)$ & $2(5.12 \%)$ & 0.001 \\
\hline Serious arrhythmias & $3(08.10 \%)$ & $2(12.82 \%)$ & $\mathrm{NA}$ \\
\hline $\begin{array}{c}\text { Recurrent non-fatal } \\
\text { MI }\end{array}$ & $2(5.40 \%)$ & 0 & 0.01 \\
\hline Recurrent angina & $2(5.40 \%)$ & $3(07.69 \%)$ & 0.31 \\
\hline $\begin{array}{c}\text { Table 3. Showing Incidence of In-Hospital MACE among } \\
\text { STEMI and UA/NSTEMI Groups Surviving the Admission } \\
\text { Event }\end{array}$ \\
\hline
\end{tabular}

(MACE, Major Acute Cardiac Events; NSTEMI, Non STElevation Myocardial Infarction; STEMI, ST-Elevation Myocardial Infarction; UA, Unstable Angina).

The overall 30-day follow-up results of patients surviving the in-hospital course displayed a significantly higher incidence of mortality in the STEMI group (2/37 - 5.40\%) compared with the UA/NSTEMI group $(0 / 39,0 \% ; p<0.001)$. Recurrent non-fatal MI was also significantly higher in the STEMI group $(2 / 37,2.70 \%)$ compared with the UA/NSTEMI group $(0 / 39,0 \% ; p<0.01)$.

\section{DISCUSSION}

Among the 76 patients admitted with ACS in the present study, $46.68 \%$ were for STEMI and $51.31 \%$ for NSTEMI. The distribution is not so similar to the GRACE registry data, which shows 32\% STEMI and 27\% for NSTEMI.13 The French registry of ACS (ONACI) shows 31\% STEMI and 29\% NSTEMI. ${ }^{14}$ Male patients constituted more than half of the present studied sample (78\%). This was similar to most of the recent studies supporting that male gender increases the risk of developing ACS.9,10,13,14 The mean age of patients in the present study was approximately $55 \pm 3.76$ and $57 \pm 4.12$ in STEMI and UA/STEMI groups respectively, while when compared with the Western data the mean age was approximately 66 years.9,10,13,14 However, there were no baseline clinical features including incidence of diabetes mellitus, hypertension, dyslipidaemia, angina, systolic blood pressure and aspirin use in the previous week and initial serum creatinine between the sample of our study and those found in other recent studies, 9,10,13,14 ST-segment deviation at presentation was reported in $82.05 \%$ of UA/NSTEMI patients. The reported rates of ST deviation at presentation varied much in the different reports being 55.9\%11 45\% $47 \%, 1532 \%-43 \%, 1233.7 \%{ }^{16}$ and $26 \% 10 ; 75.67 \%$ of the STEMI patients reached the hospital after more than $4 \mathrm{~h}$ from the pain onset in the present study. The Cardiogenic shock admission was reported to be $1.2 \%{ }^{9}$ to $1.5 \%{ }^{16}$ in all ACS patients compared with our study $(16.21 \%$ in STEMI and $5.12 \%$ in UA/NSTEMI). This difference may also be due to delayed arrival at the Hospital. In the present study, STEMI was associated with a higher incidence of in-hospital MACE, in-hospital mortality, recurrent non-fatal MI, cardiogenic shock and serious arrhythmia compared with UA/NSTEMI patients. At the 30-day follow-up, the STEMI group still had a higher incidence of all-cause mortality and recurrent nonfatal MI. These findings are consistent with those of the 
GRACE study ${ }^{17}$ and ONACI registry, which reported higher inhospital complications and higher mortality in the first $24 \mathrm{~h}$ in STEMI patients. ${ }^{16}$ The present study showed $5.40 \%$ mortality in-hospital mortality among STEMI patients and no in-hospital mortality in UA/NSTEMI patients. A similar study across Europe in 2000 reported an in-hospital mortality of $5.9 \%$ among patients with acute MI, $4.3 \%$ in patients with MI evolving from UA and $1.6 \%$ in patients with UA ${ }^{18}$ Morrow et $\mathrm{al}^{4}$ found an in-hospital mortality of $12.6 \%$ in STEMI patients. Bradshaw et $\mathrm{al}^{12}$ found that the crude in-hospital mortality among the whole ACS spectrum was $11.0 \%$ overall, being $8.9 \%, 11.1 \%$ and $11.5 \%$ at small, community and teaching hospitals respectively. In this study, it was found that patients with STEMI had a higher all-cause mortality rate (5.40\%) and recurrent non-fatal MI (51.35\%) at 30 days compared with UA/NSTEMI patients ( $0 \%$ and $20.51 \%$, respectively). In a large validation study by Morrow et al,4 TIMI score recruiting 84,029 patients with STEMI reported a 30-day mortality of $5.7 \%$. In another small single-centre retrospective study of 460 consecutive patients reported a 30-day all-cause mortality rate of $2.8 \%$ and a recurrent non-fatal MI rate of $5.2 \%{ }^{11}$ STEMI was associated with a higher risk of shortterm mortality compared with UA and NSTEMI in the ONACI registry. ${ }^{14}$ Sangu et al reported that STEMI was an independent predictor of re-hospitalisation within 30 days after an ACS.15 In addition, data from the United Kingdom indicated that the presence of MI was associated with a higher 30 -day mortality rate being $4.5 \%, 10.4 \%$ and $12.9 \%$ in the UA, NSTEMI and STEMI groups, respectively. 19 Originally, the GRACE risk score was designed to predict all-cause mortality and the TIMI risk score was designed to predict the composite endpoint of death and myocardial re-infarction. In the present study, both scores were valid in the patients and successfully predicted in-hospital MACE, 30-day all-cause mortality and 30-day recurrent non-fatal MI with a good discriminative ability in both STEMI and UA/NSTEMI patients.

\section{CONCLUSION}

The age of occurrence of ACS was relatively younger in this part of Kerala, who share common known risk factors of Coronary Artery Disease. Patients grouped in STEMI represented approximately half of ACS patients and are associated with worse in-hospital as well as 30-day outcomes. Both TIMI and GRACE risk scores are valid for use for ACS patients in this Hospital with a better discriminative ability for the GRACE score, especially in UA/STEMI patients.

\section{REFERENCES}

[1] Bassand JP, Hamm CW, Ardissino D, et al. Guidelines for the diagnosis and treatment of non-ST-segment elevation acute coronary syndromes. Eur Heart J 2007 28(13):1598-660.

[2] Wright RS, Anderson JL, Adams CD, et al. 2011 ACCF/AHA focused update incorporated into the ACC/AHA 2007 guidelines for the management of patients with unstable Angina/Non-ST-Elevation Myocardial Infarction: a report of the American College of Cardiology Foundation/American Heart Association Task Force on practice guidelines developed in collaboration with the American academy of family physicians, society for cardiovascular angiography and interventions, and the society of thoracic surgeons. J Am Coll Cardiol 2011;57(19):215-367.

[3] Antman EM, Cohen M, Bernink PJ, et al. The TIMI risk score for unstable angina/non-ST elevation MI: a method for prognostication and therapeutic decision making. JAMA 2000;284(7):835-42.

[4] Morrow DA, Antman EM, Charlesworth A, et al. TIMI risk score for ST-elevation myocardial infarction: a convenient, bedside, clinical score for risk assessment at presentation: an intravenous nPA for treatment of infarcting myocardium early II trial sub study. Circulation 2000;102(17):2031-7.

[5] Boersma E, Pieper KS, Steyerberg EW, et al. Predictors of outcome in patients with acute coronary syndromes without persistent ST-segment elevation. Results from an international trial of 9461 patients. The PURSUIT investigators. Circulation 2000;101(22):2557-67.

[6] Califf RM, Pieper KS, Lee KL, et al. Prediction of 1-year survival after thrombolysis for acute myocardial infarction in the global utilization of streptokinase and TPA for occluded coronary arteries trial. Circulation 2000;101(19):2231-8.

[7] Jacobs DR, Kroenke C, Crow R, et al. PREDICT: a simple risk score for clinical severity and long-term prognosis after hospitalization for acute myocardial infarction or unstable angina: the Minnesota heart survey. Circulation1999;100(6):599-607.

[8] Krumholz HM, Chen J, Chen YT, et al. Predicting oneyear mortality among elderly survivors of hospitalization for an acute myocardial infarction: results from the cooperative cardiovascular project. J Am Coll Cardiol 2001;38(2):453-9.

[9] Eagle KA, Lim MJ, Dabbous $\mathrm{OH}$, et al. A validated prediction model for all forms of acute coronary syndrome: estimating the risk of 6-month postdischarge death in an international registry. JAMA 2004;291(22):2727-33.

[10] Yan AT, Yan RT, Tan M, et al. Risk scores for risk stratification in acute coronary syndromes: useful but simpler is not necessarily better. Eur Heart J 2007;28(9):1072-8.

[11] Taylor J. 2012 ESC Guidelines on acute myocardial infarction (STEMI). Eur Heart J 2012;33(20):2501-2.

[12] Myocardial infarction redefined-a consensus document of The Joint European Society of Cardiology/American College of Cardiology Committee for the redefinition of myocardial infarction. Eur Heart J 2007;21(18):150213.

[13] Fox KA, Eagle KA, Gore JM, et al. The global registry of acute coronary events, 1999 to 2009-GRACE. Heart 2010;96(14):1095-101.

[14] Donataccio MP, Puymirat E, Vassanelli C, et al. Presentation and revascularization patterns of patients admitted for acute coronary syndromes in France between 2004 and 2008 (from the National Observational Study of Diagnostic and Interventional Cardiac Catheterization ONACI). Am J Cardiol 2014 113(2):243-8. 
[15] Sangu PV, Ranasinghe I, Costa AB, et al. Trends and predictors of rehospitalisation following an acute coronary syndrome: report from the Australian and New Zealand population of the Global Registry of Acute Coronary Events (GRACE). Heart 2012;98(23):1728-31.

[16] Granger CB, Goldberg RJ, Dabbous O, et al. Predictors of hospital mortality in the global registry of acute coronary events. Arch Intern Med 2003;163(19):234553.

[17] Scirica BM, Cannon CP, Antman EM, et al. Validation of the thrombolysis in myocardial infarction (TIMI) risk score for unstable angina pectoris and non-ST-elevation myocardial infarction in the TIMI III registry. Am J Cardiol 2002;90(3):303-5.
[18] Bradshaw PJ, Ko DT, Newman AM, et al. Validity of the GRACE (Global Registry of Acute Coronary Events) acute coronary syndrome prediction model for six month post-discharge death in an independent data set. Heart 2006;92(7):905-9.

[19] Das R, Kilcullen N, Morrell C, et al. The British Cardiac Society Working Group definition of myocardial infarction: implications for practice. Heart 2006;92(1):21-6. 